\title{
Сорбционные свойства церийсодержащих слоистых двойных гидроксидов
}

\author{
(C) 2021 Рыльцова И.Г., Головин С.Н., Япрынцев М.Н., Лебедева О.Е. \\ Белгородский государственный национальный исследовательский университет, Белгород
}

Поступила в редакцию 9.10.2020 г.

DOI: $10.17308 /$ sorpchrom.2021.21/3211

Объектом данного исследования являются слоистые двойные гидроксиды (СДГ) с катионным составом $\mathrm{M}^{2+}{ }_{3} \mathrm{Al}_{1-\mathrm{x}} \mathrm{Ce}_{\mathrm{x}}$, где двухзарядный катион представлен никелем или кобальтом. Для сравнения использованы образцы близкого катионного состава, не содержащие церий. Проведение синтеза методом соосаждения с последующей гидротермальной обработкой позволило получить однофазные хорошо окристаллизованные СДГ со структурой гидроталькита, о чем свидетельствуют данные рентгенофазового анализа.

Основной целью данного исследования являлось изучение влияние введения крупного катиона церия в структуру бруситоподобных слоев СДГ на сорбционные характеристики указанных материалов. Методом низкотемпературной адсорбции-термодесорбции азота по БЭТ установлено, что на площадь удельной поверхности синтезированных материалов существенное влияние оказывает катионный состав СДГ. Никельсодержащие СДГ обладают более развитой поверхностью по сравнению с кобальтсодержащими. Изотермы адсорбции-термодесорбции азота относятся к II типу по классификации БДДТ и характерны для непористых или макропористых тел.

Сорбционная способность СДГ по отношению к анионному красителю конго красному определялась статическим методом. Концентрацию красителей в растворах определяли спектрофотометрическим методом. Экспериментальные данные кинетических исследований сорбции красителя на СДГ были проанализированы с использованием моделей псевдо-первого (модель Лагергрена) и псевдо-второго (модель Хо и Маккея) порядков. Установлено, что сорбция красителя на всех синтезированных образцах СДГ адекватно описывается моделью псевдо-второго порядка.

Экспериментальные данные по равновесной адсорбции красителя на СДГ проанализировали с помощью широко используемых моделей изотерм Фрейндлиха и Ленгмюра. Показано, что изотермы сорбции для всех образцов можно отнести к типу L и в исследуемом концентрационном интервале они удовлетворительно описываются моделью Ленгмюра. На изотермах сорбции наблюдается перегиб, наличие которого может быть обусловлено переориентацией адсорбированных частиц относительно поверхности сорбента или сменой механизма сорбции с поверхностного на интеркаляционный. Установлено, что ведение крупного катиона церия в структуру бруситоподобных слоев СДГ приводит к существенному повышению сорбционной емкости указанных материалов по отношению к анионному красителю, величина максимальной адсорбции, рассчитанная по уравнению Ленгмюра в 1,4 - 2,3 раза выше для церийсодержащих СДГ. ниях СДГ.

Полученные результаты могут быть применены в сорбционных и каталитических исследова-

Ключевые слова: слоистые двойные гидроксиды, никель, кобальт, церий, адсорбциятермодесорбция азота, адсорбция конго красного

\section{Введение}

Слоистые двойные гидроксиды (СДГ) обладают двумерной структурой, состо- ящей из металлгидроксидных слоев, и способных к обмену анионов в межслоевом пространстве [1,2]. Одним из уникальных свойств материалов на основе 
СДГ является высокая сорбционная способность по отношению к органическим анионам [3-8]. СДГ также перспективны в области гетерогенного катализа и находят применение в качестве катализаторов, прекурсоров или носителей катализаторов [9-10]. В последние годы растет интерес к СДГ, содержащим редкоземельные элементы в своем составе. Этот интерес обусловлен структурными особенностями СДГ. Как известно, соединения редкоземельных элементов обладают специфическими каталитическими и фотокаталитическими свойствами, при этом на проявление указанных свойств существенное влияние оказывает структурные характеристики как активного компонента, так и матрицы $[11,12]$. Зачастую каталитическая активность напрямую зависит от сорбционных характеристик материала [13]. Интеркаляция крупного катиона церия в бруситоподобный слой может неоднозначно сказаться на сорбционной способности слоистых двойных гидроксидов: в зависимости от локализации и распределения катионов церия возможно как увеличение размеров поверхности и пористости материала, так и снижение его диффузионной проницаемости.

Ранее нами была показана принципиальная возможность синтеза гидроталькитоподобных СДГ с общей формулой $\left(\mathrm{M}^{2+}\right)_{6}\left(\mathrm{M}^{3+}\right)_{2}(\mathrm{OH})_{16} \mathrm{CO}_{3} \cdot \mathrm{nH}_{2} \mathrm{O}$, в которых в качестве двухзарядных катионов $\left(\mathrm{M}^{2+}\right)$ выступают катионы никеля, а в качестве трехзарядных $\left(\mathrm{M}^{3+}\right)$ одновременно присутствуют катионы алюминия и церия [14-15]. Настоящее исследование посвящено изучению влияния катионов церия на сорбционные характеристики никельалюминиевых и кобальт-алюминиевых СДГ.

\section{Экспериментальная часть}

Синтез. В качестве источников катионов металлов для получения СДГ использовались кристаллогидраты нитратов никеля, кобальта, алюминия и церия (III). Во всех синтезах задавалось моль- ное отношение $\mathrm{M}^{2+} /[\mathrm{Al}+\mathrm{Ce}]=3, \quad$ где $\mathrm{M}$ - Со или $\mathrm{Ni}$; мольное отношение $\mathrm{Ce} /[\mathrm{Al}+\mathrm{Ce}]$ варьировалось от 0 до 0.05 . Суммарная концентрация катионов никеля, алюминия и церия в исходном растворе составляла 1 моль/дм³. Далее в работе используются следующие сокращенные обозначения $\mathrm{NiAl}, \mathrm{CoAl}, \mathrm{CoAlCe}$ и NiAlCe, иллюстрирующие катионный состав образцов.

Соосаждение компонентов из растворов осуществляли при комнатной температуре путем добавления к исходному раствору при постоянном перемешивании по каплям раствора осадителя (смесь растворов $\mathrm{NaOH}$ и $\mathrm{Na}_{2} \mathrm{CO}_{3}$ с мольным отношением реагентов 8:1) до достижения значения $\mathrm{pH}=12$. Полученный осадок подвергали гидротермальной обработке. В случае синтеза CoAlCeСДГ гидротермальною обработку проводили при $120^{\circ} \mathrm{C}$ в течении 48 часов. В случае синтеза NiAl, CoAl и NiAlCe гидротермальною обработку проводили при $200^{\circ} \mathrm{C}$ в течении 3 часов. После охлаждения реактора полученные осадки отделяли от маточного раствора, затем многократно промывали дистиллированной водой, после чего высушивали при температуре $110^{\circ} \mathrm{C}$.

Физико-химическая характеристика. Рентгенофазовый анализ (РФА) выполняли с использованием рентгеновского дифрактометра Rigaku Ultima IV $(\mathrm{CuK} \alpha$ -излучение). Диапазон съемки 5-75 град. $2 \theta$, шаг 0.02 град., скорость 2 град/мин.

Содержание катионов металлов в синтезированных образцах определяли с использованием энергодисперсионного анализатора сканирующего электронного микроскопа QUANTA 3D при рабочем напряжении 20 кB.

Текстурные характеристики СДГ (площадь удельной поверхности и общий объем пор) определяли методом низкотемпературной адсорбциитермодесорбции азота на анализаторе удельной поверхности и пористости TriStar 3020 при температуре $77 \mathrm{~K}$. 
Сорбционные свойства СДГ изучали по отношению к анионному красителю конго красному. Для получения кинетических кривых сорбции красителя серию навесок синтезированного материала по 0.025 г заливали $25 \mathrm{~cm}^{3}$ раствора красителя с начальной концентрацией 0.05 ммоль/дм ${ }^{3}$ и выдерживали в течение заданных промежутков времени. Изучение сорбционного равновесия осуществляли статическим методом. К 0.025 г образца добавляли $25 \mathrm{~cm}^{3}$ раствора красителя с заданной концентрацией, тщательно перемешивали и выдерживали в течение 6 часов. Остаточную концентрацию красителя в растворах определяли спектрофотометрически, предварительно отделив раствор от сорбента центрифугированием. Регистрацию оптической плотности растворов осуществляли при длине волны 500 нм на спектрофотометpe SPECORD 210 Plus. Все адсорбционные эксперименты выполняли при температуре $25 \pm 1^{\circ} \mathrm{C}$.

\section{Обсуждение результатов}

По данным рентгенофазового анализа все синтезированные образцы являются однофазными и представляют собой слоистые двойные гидроксиды со структурой гидроталькита $[1,2]$. Значения параметра $c$ кристаллической решетки синтезированных СДГ, рассчитанные на основании данных РФА, представлены в таблице 1. Наблюдаются значимые отличия в величине параметра, особенно заметные для кобальт-цериевого образца. Параметр $c$ характеризует расстояние между соседними металлгидроксидными слоями, а именно равен утроенной сумме толщины бруситоподобного слоя и межслоевого расстояния, и рассчитывается как утроенное межплоскостное расстояние для рефлекса, отвечающего плоскости (003) [1,16]. Следует отметить, что величина параметра $c$ опосредованно влияет на адсорбционную способность СДГ. Так как для СДГ характерна способность к ионному обмену, то одним из механизмов поглощения ионов данными материалами является интеркаляционный. В этом случае очевидно, что анионы красителя будут легче проникать в пространство большего размера. Однако на ионообменные свойства влияет не только параметр $c$, но и природа межслоевых анионов и анионов, присутствующих в растворе $[17,18]$.

В свою очередь, параметр $c$ зависит от катионного состава СДГ. Наиболее информативной характеристикой состава является не содержание индивидуальных катионов, а отношение $\mathrm{M}^{2+} / \mathrm{M}^{3+}$, поскольку именно оно определяет избыточный заряд бруситоподобного слоя, число компенсирующих его анионов, электростатическое взаимодействие слоев и межслоевых компонентов. В таблице 1 представлен катионный состав синтезированных СДГ. Некоторое различие в отношениях $\mathrm{M}^{2+} / \mathrm{M}^{3+}$ может объяснить различие в значениях параметров $c$, вызванное влиянием заряда слоев на силы их взаимного отталкивания. Снижение отношения $\mathrm{M}^{2+} / \mathrm{M}^{3+}$ приводит также к росту количества анионов в межслоевом пространстве, способных к обмену. Наконец, нескомпенсированный заряд бруситоподобных слоев может оказывать влияние на их электростатические взаимодействия с заряженными частицами адсорбата.

Таблица 1. Катионный состав и структурные параметры СДГ

Table. 1. Cationic composition and structural parameters of LDH

\begin{tabular}{|l|c|c|c|c|c|c|c|c|}
\hline \multirow{2}{*}{ Образец } & \multicolumn{4}{|c|}{ Содержание катионов металлов, } & \multirow{2}{*}{$\mathrm{M}^{2+} / \mathrm{M}^{3+}$} & \multirow{2}{*}{ ат. $\AA$} & $\begin{array}{c}\mathrm{S}_{\text {уд, }} \\
\mathbf{M}^{2} / \Gamma\end{array}$ & $\begin{array}{c}\text { Средний диаметр } \\
\text { пор, } \AA\end{array}$ \\
\cline { 2 - 4 } & $\mathrm{Co}$ & $\mathrm{Ni}$ & $\mathrm{Al}$ & $\mathrm{Ce}$ & & & & \\
\hline CoAl & 0.700 & - & 0.300 & - & 2.3 & 23.11 & 10.7 & 89.5 \\
\hline CoAlCe & 0.730 & - & 0.256 & 0.014 & 2.7 & 22.72 & 16.3 & 126.5 \\
\hline NiAl & - & 0.727 & 0.273 & - & 2.7 & 23.21 & 39.3 & 120.7 \\
\hline NiAlCe & - & 0.714 & 0.274 & 0.012 & 2.5 & 23.34 & 36.5 & 143.5 \\
\hline
\end{tabular}




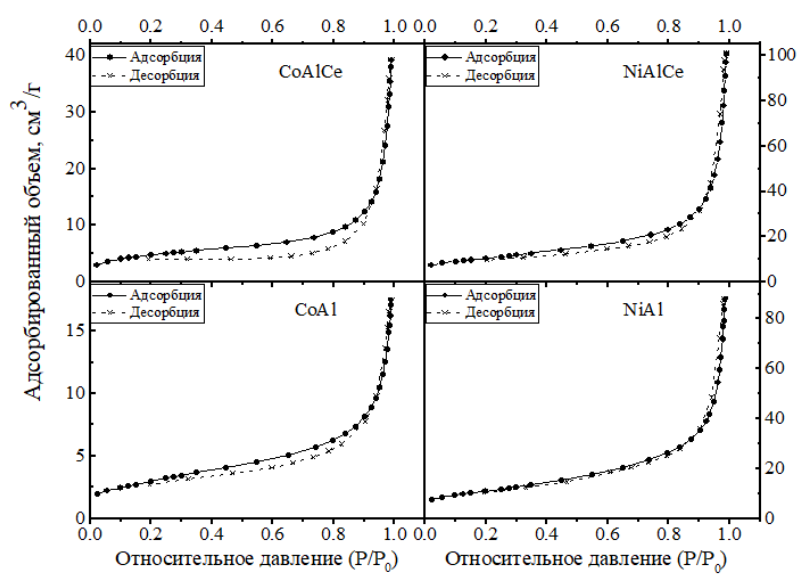

Рис.1. Изотермы адсорбции и термодесорбции азота на СДГ

Fig. 1. Isotherms of nitrogen adsorption and thermal desorption on $\mathrm{LDH}$

Изотермы низкотемпературной адсорбции-термодесорбции азота представлены на рис. 1. Для всех четырех образцов они однотипны и относятся к II типу по классификации БДДТ. Изотермы данного типа характерны для непористых или макропористых тел [19]. Площадь удельной поверхности СДГ, рассчитанная по данным адсорбциидесорбции азота в рамках теории полимолекулярной адсорбции БЭТ, и средний диаметр пор приведены в таблице 1. Относительно низкие значения площади удельной поверхности могут быть обусловлены высокой степенью кристалличности образцов, о которой свидетельствуют узкие интенсивные пики на дифрактограммах синтезированных СДГ (рис.2). Этот эффект способа получения описан для образцов, полученных в условиях гидротермальной обработки [5]. Как следует из данных таблицы 1, $\mathrm{NiAl}$ и NiAlCe СДГ обладают более раз- витой поверхностью по сравнению с CoAl и CoAlCe СДГ. Аналогичная тенденция для NiAl и CoAl приведена в работе [3].

Кинетические кривые сорбции конго красного на исследуемых образцах СДГ приведены на рис. 3. На начальном этапе, в течение 30-50 минут, для всех образцов СДГ сорбция протекает достаточно быстро, а затем замедляется, и кривые выходят на плато. Согласно общепринятому мнению, основными механизмами сорбции анионов СДГ являются ионный обмен и/или образование водородных связей с поверхностными гидроксильными группами бруситоподобных слоев $[3,17,18]$. Для оценки механизма сорбции, полученные данные были проанализированы с использованием кинетических моделей псевдо-первого (модель Лагергрена [20]) и псевдовторого (модель Хо и Маккея [21]) порядков. Рассчитанные в рамках

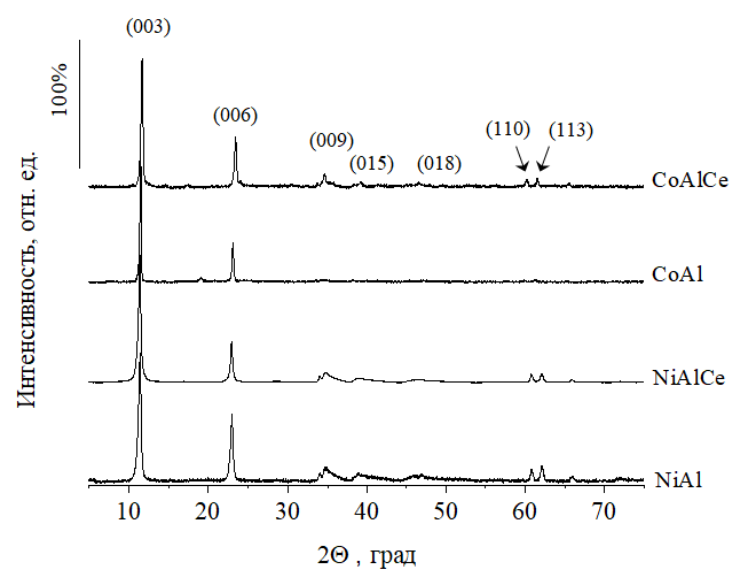

Рис.2. Дифрактограммы образцов СДГ

Fig. 2. Diffraction patterns of LDH samples 
Таблица 2. Рассчитанные в рамках кинетических моделей параметры сорбции красителя конго красного на СДГ

Table 2. The parameters of the sorption of Congo red dye on LDH calculated using kinetic models

\begin{tabular}{|c|c|c|c|c|}
\hline Образец & CoAl & CoAlCe & $\mathrm{NiAl}$ & NiAlCe \\
\hline $\begin{array}{c}\mathrm{A}_{\mathrm{e}},(\text { ммоль/г) } \\
\text { экспериментальное }\end{array}$ & 0.0231 & 0.0289 & 0.0388 & 0.0512 \\
\hline \multicolumn{5}{|c|}{ Кинетическая модель псевдо-первого порядка } \\
\hline $\mathrm{K}_{1}$ & 0.0095 & 0.0064 & 0.0202 & 0.0154 \\
\hline $\begin{array}{c}\mathrm{A}_{\mathrm{e}},(\text { ммоль } / г) \\
\text { расчётное }\end{array}$ & 0.0123 & 0.0173 & 0.0187 & 0.0167 \\
\hline $\mathrm{R}^{2}$ & 0.8071 & 0.9379 & 0.9052 & 0.8300 \\
\hline \multicolumn{5}{|c|}{ Кинетическая модель псевдо-второго порядка } \\
\hline $\mathrm{K}_{2}$ & 2.314 & 1.0213 & 3.350 & 3.889 \\
\hline $\begin{array}{c}\mathrm{A}_{\mathrm{e}},(\text { (ммоль/г) } \\
\text { расчётное }\end{array}$ & 0.0235 & 0.0297 & 0.0398 & 0.0517 \\
\hline $\mathrm{R}^{2}$ & 0.9920 & 0.9929 & 0.9997 & 0.9996 \\
\hline
\end{tabular}

указанных кинетических моделей параметры приведены в табл. 2. Можно видеть, что сорбция красителя конго красного на всех синтезированных СДГ более адекватно описывается кинетическим уравнением псевдо-второго порядка Хо и Маккея, о чем свидетельствуют хорошее совпадение расчётных и экспериментальных величин Ае и значения коэффициентов корреляции $\mathrm{R}^{2}>0.99$. Это позволяет сделать заключение о преобладании вклада хемосорбции в скорость процесса $[3,6]$.

На рис. 4 представлены изотермы сорбции конго красного на СДГ. По виду их можно отнести к типу L классификации по Гильсу [22]. Этот тип характерен

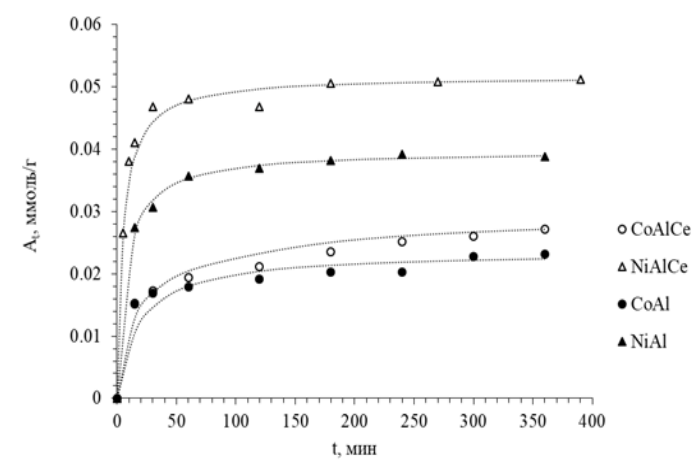

Рис. 3. Кинетические кривые сорбции на СДГ красителя конго красного из растворов с концентрацией 0.05 ммоль/дм ${ }^{3}$

Fig. 3. Sorption kinetic curves of congo red dye on LDH from solutions with a concentration of $0.05 \mathrm{mmol} / \mathrm{dm}^{3}$ для случаев высокой степени сродства между поверхностью адсорбента и молекулами красителя. На изотермах сорбции заметен перегиб при равновесной концентрации красителя выше 0.04 ммоль/дм², особенно сильно проявляющийся для содержащих церий образцов. Подобный вид изотерм адсорбции встречается в работах, посвященных сорбции красителей на СДГ $[3,7,8]$. Наличие перегиба на изотермах может быть обусловлено переориентацией адсорбированных частиц относительно поверхности сорбента или сменой механизма сорбции с поверхностного на интеркаляционный.

Как следует из представленных на

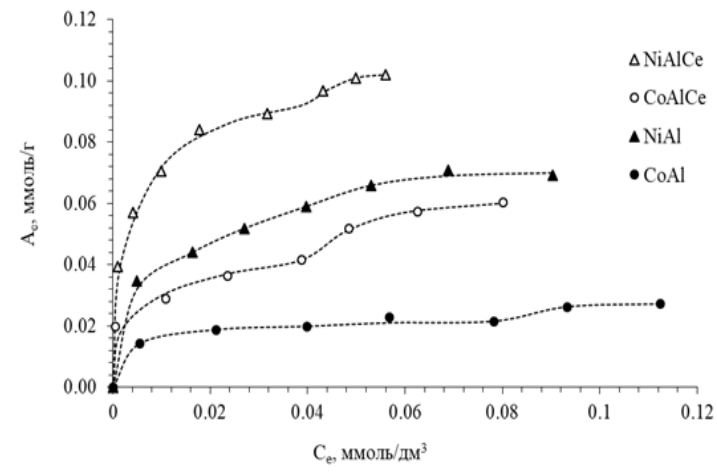

Рис. 4. Изотермы сорбции красителя конго красного на СДГ

Fig. 4. Sorption isotherms of Congo red dye on $\mathrm{LDH}$ 
Таблица 3. Параметры изотермы сорбции красителя конго красного на СДГ

Table 3. Parameters of the sorption isotherm of the Congo red dye on LDH

\begin{tabular}{|c|c|c|c|c|}
\hline Образец & СоAl & СоАІСе & NiAl & NiAlCe \\
\hline \multicolumn{5}{|c|}{ Модель Фрейндлиха } \\
\hline $\mathrm{k}_{\mathrm{F}},($ ммоль/г) & 0.0399 & 0.0919 & 0.1359 & 0.2087 \\
\hline $1 / \mathrm{n}$ & 0.1996 & 0.2138 & 0.2608 & 0.2386 \\
\hline $\mathrm{R}^{2}$ & 0.9271 & 0.8916 & 0.9752 & 0.9930 \\
\hline \multicolumn{5}{|c|}{ Модель Ленгмюра } \\
\hline $\mathrm{k}_{\mathrm{L},(\text { дм }}^{3}$ мммоль) & 84.35 & 81.82 & 96.57 & 251.46 \\
\hline $\begin{array}{c}\mathrm{A}_{\infty,}(\text { ммоль/г) } \\
\text { рассчетное }\end{array}$ & 0.0283 & 0.0656 & 0.0778 & 0,1069 \\
\hline $\mathrm{R}^{2}$ & 0.9676 & 0,9392 & 0.9904 & 0.9954 \\
\hline
\end{tabular}

рис. 4 данных, сорбционная емкость образцов, содержащих никель в качестве двухзарядного катиона, превышает сорбционную способность образцов, содержащих кобальт, что согласуется с данными об удельной поверхности образцов и отношении $\mathrm{M}^{2+} / \mathrm{M}^{3+}$ (табл. 1). При сравнении образцов, имеющих в составе церий, и синтезированных без церия наблюдается значительное увеличение сорбционной емкости по отношению к красителю конго красному в случае содержащих церий образцов. Это позволяет сделать вывод о благоприятном влиянии введения церия в структуру бруситоподобных слоев на сорбционную способность СДГ.

Экспериментальные данные по равновесной адсорбции были проанализированы с помощью широко используемых моделей изотерм Фрейндлиха и Ленгмюра. Параметры, рассчитанные в рамках двух указанных моделей, представлены в таблице 3.

Анализ полученных данных показывает, что сорбция красителя конго крас- ного лучше описывается моделью Ленгмюра, коэффициенты корреляции $\mathrm{R}^{2}$ для этой модели выше. Сопоставляя рассчитанные значения предельных адсорбции для NiAl c NiAlCe и CoAl c CoAlCe СДГ, можно отметить, что величина $\mathrm{A}_{\infty}$ возрастает при ведении катиона редкоземельного элемента в состав бруситоподобных слоев. Сравнение рассчитанных по уравнению Ленгмюра значений предельной сорбции с литературными данными позволяет заключить, что синтезированные материалы демонстрируют вполне типичные для данного класса соединений сорбционные характеристики по отношению к красителю конго красному [7,8,23].

\section{Заключение}

Таким образом, из представленных данных следует, что введение церия в структуру СДГ существенным образом не влияет на скорость процесса сорбции, но приводит к повышению сорбционной емкости указанных материалов по отношению к анионному красителю.

\section{Работа выполнена с использованием научного оборудования ЦКП «Технологии и Материаль НИУ «БелГУ» при поддержке гранта РФФИ № 18-43-310011 $p$ a.}

\section{Список литературы}

1. Cavani F., Trifiro F., Vaccari A. //Catal. Today. 1991. Vol. 11. pp. 173-301. DOI: http://dx.doi.org/10.1016/0920-5861(91)80068$\mathrm{K}$.

2. Forano C., Hibino T., Leroux F., Taviot-
Guého C. Developments in Clay Science: Handbook of Clay Science / Faïza Bergaya, Benny K.G. Theng, Gerhard Lagaly, Elsevier. 2006. Vol. 1. pp. 1021-1095. DOI: https://doi.org/10.1016/S1572-4352(05)01039-1.

3. Zhang C., Yang S., Chen H., He H. et al. // Appl. Surf. Sci. 2014. Vol. 301. pp. 329-337. 
DOI: https://doi.org/10.1016/j.apsusc.2014.02. 073.

4. Chilukoti S., Thangavel T. // Inorg. Chem. Commun. 2019. Vol. 100. pp. 107-117. DOI: https://doi.org/10.1016/j.inoche.2018.12.027.

5. Extremera R., Pavlovic I., Pérez M.R., Barriga C. // Chem. Eng. J. 2012. Vol. 213. pp. 392-400. DOI: https://doi.org/10.1016/j.cej. 2012.10.042.

6. Ahmed I.M., Gasser M.S. // Appl. Surf. Sci. 2012. Vol. 259. pp. 650-656. DOI: https:// doi.org/10.1016/j.apsusc.2012.07.092.

7. Shan R., Yan L., Yang Y., Yang K. et al. // J. Ind. Eng. Chem. 2015. Vol. 21. pp. 561-568. DOI: https://doi.org/10.1016/j.jiec.2014.03.019.

8. Lafi R., Charradi K., Djebbi M.A., Ben Haj Amara A. et al. // Adv. Powder Technol. 2016. Vol. 27. No 1. pp. 232-237. DOI: https://doi.org/10.1016/j.apt.2015.12.004.

9. Fan G., Li F., Evans D.G., Duan X. // Chem. Soc. Rev. 2014. Vol. 43. pp. 7040-7066 https://doi.org/10.1039/C4CS00160E.

10.He S., An Z., Wei M., Evans D.G. et al. // Chem. Commun. 2013. Vol. 49. pp. 5912-5920. DOI: https://doi.org/10.1039/C3CC42137F.

11.Montini T., Melchionna M., Monai M., Fornasiero P. // Chem. Rev. 2016. Vol. 116. No 10. pp. 5987-6041. DOI: https://doi.org/10.1021 /acs.chemrev.5b00603.

12.Гурин В.С., Бобкова Н.М., Трусова Е.Е. // Химия в интересах устойчивого развития. 2015. T. 23. C. 25-31.

13.Bouberka Z., Benabbou K.A., Khenifi A., Maschke U. // J. Photochem. Photobiol. A. 2014. Vol. 275. pp. 21-29. DOI: https://doi. org/10.1016/j.jphotochem.2013.10.010.

14.Lebedeva O.E., Ryl'tsova I.G., Yapryntsev
M.N., Golovin S.N., et al. // Pet. Chem. 2019. Vol. 59. No 7. pp. 751-755. DOI: https:/ doi.org/10.1134/S0965544119070089.

15.Golovin S.N., Yapryntsev M.N., Ryltsova I.G., Veligzhanin A.A. et al. // Chem. Pap. 2020. Vol. 74. No. 1. pp. 367-370. DOI: https://doi.org/10.1007/s11696-019-00877-9.

16.Evans D.G., Slade R.C.T. 2006. "Structural Aspects of Layered Double Hydroxides" in Layered Double Hydroxides. Structure and Bonding, Duan X., Evans D.G., eds,. Springer, Berlin. Heidelberg. Vol. 119. pp. 1-87. DOI: https://doi.org/10.1007/430_005.

17.Meyn M., Beneke K., Lagaly G. // Inorg. Chem. 1990. Vol. 29. pp. 5201-5207. DOI: https://doi.org/10.1021/ic00351a013.

18. Miyata S. // Clays Clay Miner. 1983. Vol. 31. No 4. pp. 305-311. DOI: https://doi.org/ 10.1346/CCMN.1983.0310409.

19.Грег С., Синг К. Адсорбичи, удельная поверхность, пористость. М.Мир. $1984.306 \mathrm{c}$.

20.Lagergren S. About the theory of so-called adsorption of soluble substance. Kungliga Svenska Vetenskapsakademiens. Handlingar. 1898. Vol. 21. pp. 1-39.

21.Ho Y.S., McKay G. // Process Biochemistry. 1999. Vol. 34. Is. 5.pp. 451-465. DOI: https://doi.org/10.1016/S0032-9592(98)00112-5.

22.Giles C.H., MacEwan T.H., Nakhwa S.N., Smith D. // J. Chem. Soc. 1960. pp. 3973-3993.

23.Bharali D., Deka R.C. // J. Environ. Chem. Eng. 2017. Vol. 5. No 2. pp. 2056-2067. DOI: https://doi.org/10.1016/j.jece.2017.04.012.

\title{
Sorption properties of cerium-containing layered double hydroxides
}

\author{
(C) 2021 Ryltsova I.G., Golovin S.N., Yaprintsev M.N., Lebedeva O.E. \\ Belgorod State National Research University, Belgorod, Russian Federation
}

The object of this study was layered double hydroxides $(\mathrm{LDH})$ with a cationic composition $\mathrm{M}^{2+}{ }_{3} \mathrm{Al}_{1-}$ ${ }_{\mathrm{x}} \mathrm{Ce}_{\mathrm{x}}$ where the doubly charged cation is nickel or cobalt. For comparison, we used samples of a similar cationic composition that does not contain cerium. Synthesis by coprecipitation followed by hydrothermal treatment allowed obtaining single-phase well-crystallized LDH with a hydrotalcite structure, as was evidenced by the data from an X-ray phase analysis.

The main goal of this study was the investigation of the effect of the introduction of a large cerium cation into the structure of brucite-like layers of LDH on the sorption characteristics of these materials. As was shown using low-temperature adsorption-thermal desorption of nitrogen according to BET the method, the cationic composition of LDH had a significant effect on the specific surface area of the synthesized materials. Nickel-containing LDH have a more developed surface than cobalt-containing ones. The nitrogen ad- 
sorption-thermal desorption isotherms are of type II according to the classification of BDDT and are characteristic of non-porous or macroporous bodies.

The sorption capacity of LDH in relation to the Congo red anionic dye was determined by the static method. The concentration of dyes in solutions was determined spectrophotometrically. Experimental data of kinetic studies of dye sorption on LDH were analysed using pseudo-first (Laguerin's model) and pseudosecond (Ho and McKay's model) orders. It was found that the sorption of the dye on all synthesized LDH samples was adequately described by the pseudo-second order model.

Experimental data on the equilibrium adsorption of the dye on LDH were analysed using the widely used Freundlich and Langmuir isotherm models. It was shown that sorption isotherms for all samples can be attributed to type $\mathrm{L}$ and in the studied concentration range they are satisfactorily described by the Langmuir model. An inflection was observed on the sorption isotherms, the presence of which may be due to the reorientation of the adsorbed particles relative to the sorbent surface or a change in the sorption mechanism from surface to intercalation mechanism. It was found that the introduction of a large cerium cation into the structure of brucite-like layers of LDH led to a significant increase in the sorption capacity of these materials with respect to the anionic dye, the maximum adsorption value calculated using the Langmuir equation was 1.4 2.3 times higher for cerium-containing LDH. The obtained results can be used in the sorption and catalytic studies of LDH.

Keywords: layered double hydroxides, nickel, cobalt, cerium, nitrogen adsorption-thermal desorption, Congo red dye adsorption.

\section{References}

1. Cavani F., Trifiro F., Vaccari A., Catal. Today, 1991, Vol. 11, pp. 173-301. DOI: http://dx.doi.org/10.1016/0920-5861(91)80068-K.

2. Forano C., Hibino T., Leroux F., TaviotGuého C. Developments in Clay Science: Handbook of Clay Science / Faïza Bergaya, Benny K.G. Theng, Gerhard Lagaly, Elsevier, 2006, Vol. 1, pp. 1021-1095. DOI: https://doi. org/10.1016/S1572-4352(05)01039-1.

3. Zhang C., Yang S., Chen H., He H. et al., Appl. Surf. Sci., 2014, Vol. 301, pp. 329-337. DOI: https://doi.org/10.1016/j.apsusc.2014.02.073.

4. Chilukoti S., Thangavel T., Inorg. Chem. Commun., 2019, Vol. 100, pp. 107-117. DOI: https://doi.org/10.1016/j.inoche.2018.12.027.

5. Extremera R., Pavlovic I., Pérez M.R., Barriga C., Chem. Eng. J., 2012, Vol. 213, pp. 392-400. DOI: https://doi.org/10.1016/j.cej. 2012.10.042.

6. Ahmed I.M., Gasser M.S., Appl. Surf. Sci., 2012, Vol. 259, pp. 650-656. DOI: https:// doi.org/10.1016/j.apsusc.2012.07.092.

7. Shan R., Yan L., Yang Y., Yang K. et al., J. Ind. Eng. Chem., 2015, Vol. 21, pp. 561-568. DOI: https://doi.org/10.1016/j.jiec.2014.03.019.

8. Lafi R., Charradi K., Djebbi M.A., Ben Haj Amara A. et al., Adv. Powder Technol., 2016, Vol. 27, No 1, pp. 232-237. DOI: https:// doi.org/10.1016/j.apt.2015.12.004.

9. Fan G., Li F., Evans D.G., Duan X., Chem. Soc. Rev., 2014, Vol. 43, pp. 7040-7066 https://doi.org/10.1039/C4CS00160E.

10.He S., An Z., Wei M., Evans D.G. et al., Chem. Commun., 2013, Vol. 49, pp. 5912-5920.
DOI: https://doi.org/10.1039/C3CC42137F.

11.Montini T., Melchionna M., Monai M., Fornasiero P., Chem. Rev., 2016, Vol. 116, No 10, pp. 5987-6041. DOI: https://doi.org/10.1021 /acs.chemrev.5b00603.

12.Gurin V.S., Bobkova N.M., Trusova E.E., Khimiya $v$ interesakh ustoychivogo razvitiya, 2015, Vol. 23, pp. 25-31.

13.Bouberka Z., Benabbou K.A., Khenifi A., Maschke U., J. Photochem. Photobiol. A, 2014, Vol. 275, pp. 21-29. DOI: https://doi. org/10.1016/j.jphotochem.2013.10.010.

14.Lebedeva O.E., Ryl'tsova I.G., Yapryntsev M.N., Golovin S.N. et al., Pet. Chem., 2019, Vol. 59, No 7, pp. 751-755. DOI: https:/doi. org/10.1134/S0965544119070089.

15.Golovin S.N., Yapryntsev M.N., Ryltsova I.G., Veligzhanin A.A. et al., Chem. Pap., 2020, Vol. 74, No 1, pp. 367-370. DOI: https://doi.org/10.1007/s11696-019-00877-9.

16.Evans D.G., Slade R.C.T. 2006. "Structural Aspects of Layered Double Hy-droxides" in Layered Double Hydroxides. Structure and Bonding, Duan X., Evans D.G., eds,. Springer, Berlin. Heidelberg, Vol. 119, pp. 1-87. DOI: https://doi.org/10.1007/430_005.

17.Meyn M., Beneke K., Lagaly G., Inorg. Chem., 1990, Vol. 29, pp. 5201-5207. DOI: https://doi.org/10.1021/ic00351a013.

18. Miyata S., Clays Clay Miner, 1983, Vol. 31, No 4, pp. 305-311. DOI: https://doi.org/ 10.1346/CCMN.1983.0310409.

19.Greg S., Sing K. Adsorbtsiya, udel'-naya poverkhnost', poristost', M., Mir, 1984, 306 p. 
20.Lagergren S. About the theory of so-called adsorption of soluble substance. Kungliga Svenska Vetenskapsakademiens, Handlingar, 1898, Vol. 21, pp. 1-39.

21.Ho Y.S., McKay G., Process Biochemistry, 1999, Vol. 34, Is. 5, pp. 451-465. DOI: https://doi.org/10.1016/S0032-9592(98)

Рыльцова Ирина Геннадьевна - доцент кафедры общей химии, к.х.н., Белгородский государственный национальный исследовательский университет, Белгород

Головин Сергей Николаевич - аспирант кафедры общей химии, Белгородский государственный национальный исследовательский университет, Белгород

Япрынцев Максим Николаевич - научный сотрудник ЦКП «Технологии и материалы НИУ «БелГУ», к.ф.-м.н., Белгород

Лебедева Ольга Евгеньевна - заведующий кафедрой общей химии, д.Х.Н., профессор, Белгородский государственный национальный исследовательский университет, Белгород
00112-5.

22.Giles C.H., MacEwan T.H., Nakhwa S.N., Smith D., J. Chem. Soc., 1960, pp. 3973-3993.

23.Bharali D., Deka R.C., J. Environ. Chem. Eng., 2017, Vol. 5, No 2, pp. 2056-2067. DOI: https://doi.org/10.1016/j.jece.2017.04.012.

Ryltsova Irina G. - Ph.D. (chemistry), Associate Prof., Department of General Chemistry, Belgorod State National Research University, Belgorod, email: ryltsova@bsu.edu.ru

Golovin Sergei N. - PhD student, Department of General Chemistry, Belgorod State National Research University, Belgorod, e-mail: 801492@bsu.edu.ru

Yapryntsev Maksim N. - PhD (physics and math), junior researcher of the collective use center "Technologies and Materials of the National Research University BSU, Belgorod, e-mail: yaprintsev@bsu.edu.ru

Lebedeva Olga E. - Head of Department, Doctor of chemical science, professor, Department of General Chemistry, Belgorod State National Research University, Belgorod, e-mail: OLebedeva@bsu.edu.ru 